\title{
Compresión del nervio cubital en el codo: resultados del tratamiento quirúrgico con descompresión y reconstrucción de un nuevo retináculo epitrócleo-olecraneano
}

\author{
G. Celester ${ }^{1,2}$, A. Prego ${ }^{1,2}$, F. González del Castillo ${ }^{1,2}$, G. Monje 2 , \\ R. Arriaza ${ }^{2}$. \\ Unidad de Cirugía de La Mano del Hospital Modelo. ${ }^{2}$ Instituto Médico Arriaza \\ y Asociados. A Coruña. España.
}

\begin{abstract}
Resumen: Objetivo: Presentar los resultados del tratamiento quirúrgico de la compresión del nervio cubital en el codo mediante descompresión y reconstrucción de un nuevo retináculo epitrócleo-olecraneano.

Material y método: Se estudiaron 5 I codos en 49 pacientes: 25 mujeres y 24 hombres, con una edad media de 44,2 años. En todos los pacientes se anotó el aspecto macroscópico del nervio, registrándose la zona en la que éste se presentaba comprimido, así como la existencia de seudoneuroma, imagen en reloj de arena, cambios de coloración o alteraciones morfológicas de otro tipo.

En la clasificación de McGowan, I I casos presentaban un grado I, 28 casos un grado II y I 2 casos un grado III.

De los 49 pacientes, 43 estaban en edad laboral, la mayor parte en situación de baja laboral en el momento de la cirugía. Resultados: En los $5 \mathrm{I}$ casos, se obtuvo un resultado excelente en 37 (72,6\%), bueno en 10 (19,6\%) y regular en 4 (7,8\%). Todos los pacientes que se encontraban de baja laboral se reincorporaron a sus actividades laborales con una media de $4 \mathrm{I}, 5$ días.

Conclusiones: La técnica de descompresión con reconstrucción de un nuevo retináculo epitrócleo-olecraneano es una intervención quirúrgica sencilla que impide la subluxación anterior propia de la descompresión simple y evita las complicaciones de las transposiciones anteriores y de las epitroclectomías.

Esta técnica está indicada en todos los grados de afectación siempre que la anatomía del codo sea normal y en pacientes que no hayan sido operados previamente con transposiciones anteriores.
\end{abstract}

Palabras claves: Síndrome de compresión del nervio cubital, síndrome del túnel cubital, nervio cubital.

\begin{abstract}
Purpose:To describe the results of the surgical treatment of the ulnar nerve compression syndrome performing a decompression and a reconstruction of an epitrochlea-olecranon pulley.

Material and methods: $5 \mathrm{I}$ elbows were studied in 49 patients: 25 women and 24 men, with a mean age of 44,2 years. In all the patients the macrocospic aspect of the nerve was annotated and the area in which it was compressed, the existence of pseudoneuroma, hourglass aspect, changes of coloration or any morphologic alterations, registered. In McGowan's classification II cases were presenting degree I, 28 cases degree II and I 2 cases degree III. Of 49 patients, 43 were in working age, most off work sue to sickness in the moment of the surgery.

Results: In the 51 cases, an excelent result was obtained in 37 (72,6\%), good in 10 (19,6\%) and regular in 4 (7,8\%). All the patients labor fall rejoined to his activities and habitual professions.

Conclusions: The technique of decompression with reconstruction of a pulley epitrócleo-olecraneana is a simple surgical technique that prevents the anterior subluxation of the simple decompression technique and avoids the complications of both the anterior transpositions and the medial epicondylectomy.

This technique is safer than the previous transpositions as for prediction of results, is indicated in all the degrees of affectation providing that the anatomy of the elbow should be normal and in patients who were not operated on before by previous transpositions.
\end{abstract}

Key words: Ulnar Nerve Compression Syndrom, Cubital Tunnel Syndrome, Ulnar Nerve.

La compresión del nervio cubital en el codo es la segunda neuropatía por compresión más frecuente del miembro superior, después del Síndrome del Túnel Carpiano'.

A Panas $^{2}$ se le atribuye la primera referencia bibliográfica de esta neuropatía, que es, así mismo, la primera
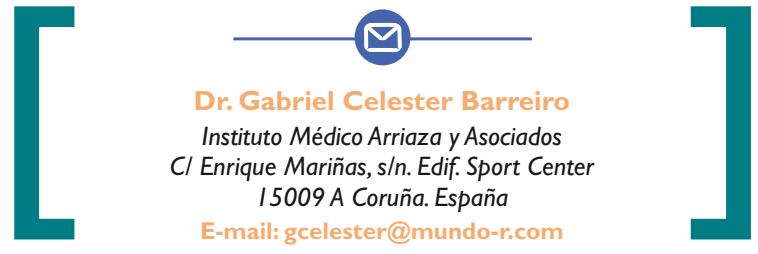
mención descrita de la afección crónica de un nervio periférico, publicada en 1878.

La literatura médica en lengua inglesa suele denominar como Síndrome del Túnel Cubital a las neuropatías por compresión de este nervio en la proximidad del codo. Dada la extensión de este concepto en la bibliografía internacional, en el presente artículo lo abreviaremos como STCub para evitar una confusión de siglas con la más habitual del Síndrome del Túnel Carpiano (STC).

Con respecto al tratamiento quirúrgico del STCub, pese a ser la segunda neuropatía más operada, también por detrás del STC, no hay un acuerdo unánime sobre la técnica de elección, siendo este asunto muy controvertido y en permanente discusión.

Los objetivos del presente trabajo son tres. El primero es la descripción de una técnica que evita la amplia disección del nervio propia de las transposiciones, así como el riesgo de subluxación recurrente de las descompresiones simples y las posibles complicaciones de las osteotomías epitrocleares (trastornos de la musculatura pronatoflexora, sangrado del lecho nervioso con ulterior fibrosis perineural, etc.). El segundo, es la descripción de los hallazgos anatómicos encontrados durante la intervención quirúrgica y el tercero, la descripción de los resultados clínicos obtenidos en 5 I neuropatías cubitales operadas con esta técnica quirúrgica.

\section{MATERIALY MÉTODO}

Se trata de un estudio retrospectivo en el que se analiza una serie personal de 5 I codos operados de STCub mediante una técnica reconstructora de un nuevo retináculo epitrócleo-olecraneano, desde el 28 de enero de 2004 hasta el 31 de diciembre de 2012 (9 años).

La edad media global de esta serie resultó de 44,2 años, con 43 pacientes (87,7\%) en edad laboral. Sólo una paciente fue menor de edad (niña de 13 años con STCub postraumático) y tres por encima de 65 años (77, 77 y 80 años), uno de ellos también de origen postraumático, postural, tras un largo período de estancia en coma en la $\mathrm{UCl}$.

Se operaron 5 I codos en 49 pacientes, 25 mujeres (51\%) y 24 hombres (49\%).

El codo izquierdo resultó operado en 36 casos (70,6\%) y el derecho en 15 (29,4\%). Dos pacientes eran zurdos: un varón operado del codo derecho y una mujer operada del codo izquierdo. En dos mujeres la intervención fue bilateral.

De los 49 pacientes operados, el 55, I\% tenía ocupaciones de poca demanda funcional, el 24,5\% baja o moderada, y sólo la quinta parte $(20,4 \%)$ tenía trabajos de grandes esfuerzos.

De los 37 pacientes asegurados por cuenta ajena, 17 provenían de una mutua de accidentes de trabajo y todos estaban en situación de baja laboral.

El diagnóstico en todos los pacientes de esta serie fue siempre clínico y, al menos, con una electromiografía (EMG) previa que confirmó el diagnóstico clínico.

La evaluación de los resultados se realizó de forma clínica empleando la clasificación de McGowan33:

Grado I: Síntomas persistentes de un síndrome irritativo con ausencia de signos objetivos y sin debilidad motora detectable.

Grado II: Parestesias o dolor en el territorio cubital de la mano con hipoestesia clara y debilidad motora. Grado III: Afectación sensitiva severa con paresia y atrofia de la musculatura intrínseca.

I I casos presentaron un grado I, 28 casos un grado II y 12 casos un grado III. En todos los pacientes con grado I hubo un tiempo prudencial de espera tras los consejos oportunos sobre medidas posturales y de apoyo del codo.

En esta serie se operó patología concomitante del mismo miembro en II pacientes: 5 epitrocleitis, 4 síndromes del túnel carpiano, I rizartrosis del pulgar, I ganglión dorsal de la muñeca.

El seguimiento medio del último examen postoperatorio fue de 3,4 años (9 meses a 8,2 años).

\section{Técnica quirúrgica y anatomía quirúrgica:}

La primera parte de la técnica quirúrgica empleada, fue la habitual para la descompresión simple del túnel cubital', 4 . Se empleó anestesia regional axilar en 46 casos (90,2\%) e intravenosa en 5 casos (9,8\%); la decisión de una u otra fue tomada por los anestesistas. Con el paciente en decúbito supino e isquemia en la zona proximal del brazo, se coloca éste sobre tabla o mesa de mano poniendo dos o tres paños bajo el codo. El cirujano se sitúa en el lado axilar del paciente con gafas lupa, y el ayudante, enfrente, sujeta al miem- 


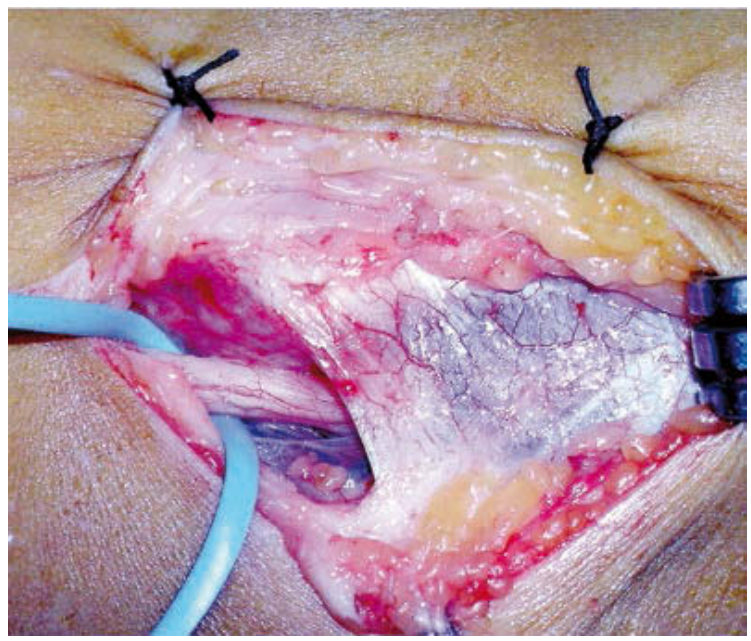

Figura I. Ligamento epitrócleo-olecraneano íntimamente adherido a la fascia del FCU, sin solución de continuidad entre ambos.

bro en rotación externa colocando el antebrazo en máxima supinación. La incisión cutánea es longitudinal sobre el canal epitrócleo-olecraneano procurando identificar y aislar la rama o ramas sensitivas del nervio antebraquial cutáneo interno (NACl) que suele cruzar, de forma oblicua con respecto a la incisión, de I a 3 $\mathrm{cm}$ distales a la epitróclea entre la zona profunda del tejido celular subcutáneo y la fascia superficial.

Seguidamente, se expone la cara superficial del túnel cubital identificando, cuando es posible, el límite entre el ligamento epitrócleo-olecraneano y el triángulo fascial de base proximal que hay entre este ligamento y las dos cabezas de inserción del Flexor Carpi Ulnaris (FCU). Esta separación no siempre está bien delimitada; en algunos pacientes no se aprecia solución de continuidad entre el ligamento y la fascia (-) Figura I porque, en realidad, esta cintilla epitrócleo-olecraneana no es un verdadero ligamento ya que no tiene las características histológicas de éstos, tratándose más bien de un refuerzo o engrosamiento de las fibras transversales de la fascia con la función de impedir los movimientos del nervio; esta cintilla, según Testut ${ }^{5}$, representaría los restos del músculo epitrocleocubital ya desaparecido en el hombre. En otros pacientes el borde inferior del ligamento está unido al puente fascial entre ambas cabezas del FCU por un arco de tejido fibroso que recuerda a la arcada de Fröhse del Supinador corto (- Figura 2. En estos casos debe hacerse una disección muy cuidadosa porque el nervio puede estar adherido a este arco fibroso.

En las raras ocasiones en que el ligamento epitrócleoolecraneano está laxo en flexión puede ser respetado,

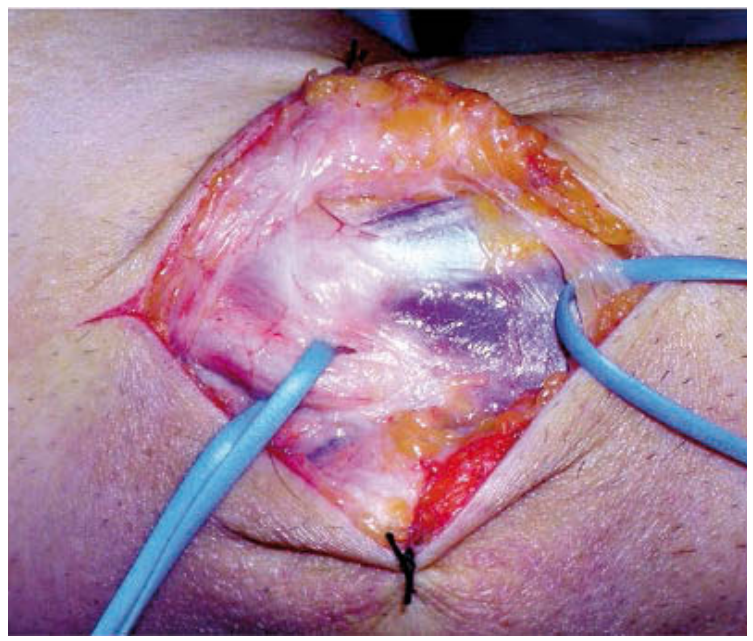

Figura. 2. En algunos pacientes el borde distal del ligamento está unido al puente fascial entre ambas cabezas del FCU por un arco de tejido fibroso que recuerda a la arcada de Fröhse del supinador corto.

ya que no está ahí el punto de compresión, debiendo buscarlo en sentido proximal y/o distal. En caso contrario, que es lo habitual, se secciona el ligamento y la fascia hasta la unión de ambas cabezas del FCU, separándolas y resecando unas fibras musculares de cualquiera de las dos cabezas para evitar ulteriores compresiones.

A continuación, se procede a la reconstrucción de un nuevo retináculo epitrócleo-olecraneano empleando una bandeleta de fascia superficial o de epimisio de la musculatura pronatoflexora insertada en la epitróclea (4) Figura 3, tallando un rectángulo de unos $15-20 \mathrm{~mm}$ de largo por 7-8 $\mathrm{mm}$ de ancho que se deja unido por su base y se gira para suturarla al otro lado del nervio. Habitualmente, esta bandeleta se talla en la cara anterior y se voltea hacia posterior, pero también se realizó de posterior a anterior (- Figura 4, y en algún caso se emplearon dos hemibandeletas suturadas sobre el nervio (1) Figura 5, una pequeña bandeleta suturada al propio ligamento epitrócleo-olecraneano conservando una de sus inserciones, o el mismo ligamento epitrócleo-olecraneano ampliando su base en sentido longitudinal (- Figura 6. El modo en que se haga es irrelevante siempre que se reconstruya un nuevo retináculo epitrócleo-olecraneano laxo en flexión pero que impida la subluxación anterior del nervio.

Con el fin de evitar la hipoestesia postquirúrgica en la cara interna del codo y el antebrazo, en todos los casos se buscó la rama dorsal del NACl que suele cruzar por delante de la epitróclea. Habitualmente es una 

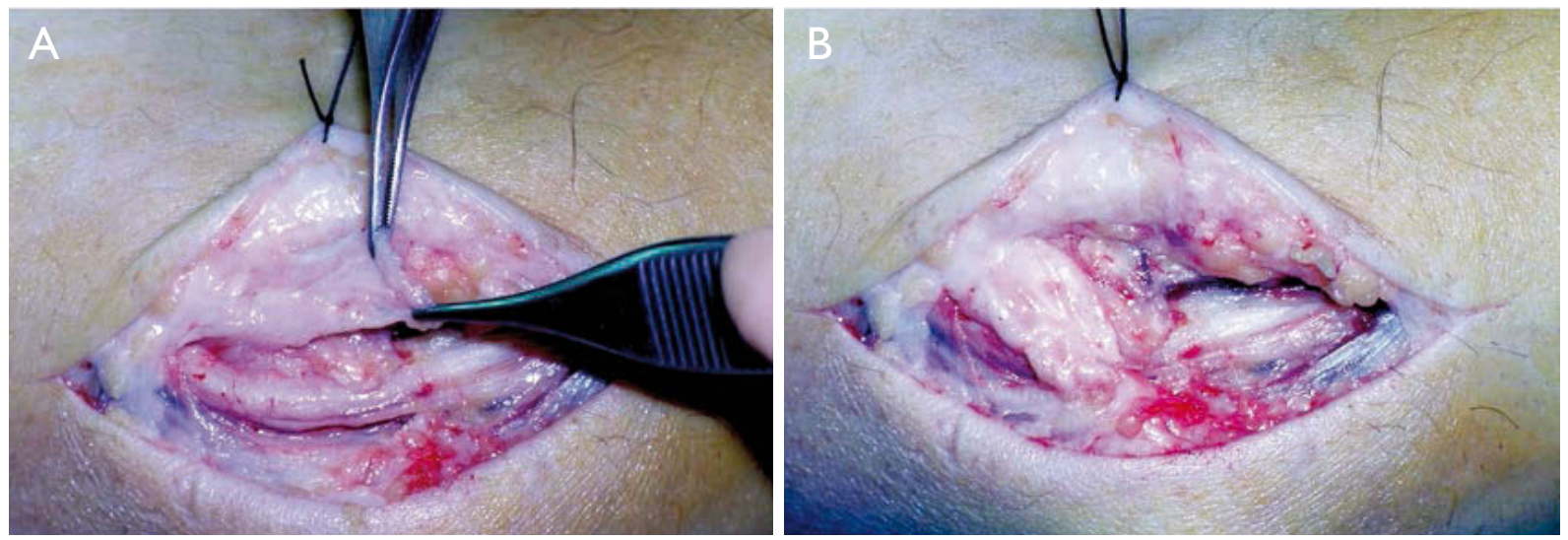

Figura 3. Reconstrucción de un nuevo retináculo epitrócleo-olecraneano $\mathbf{A}$. se emplea una bandeleta de fascia superficial de unos 15-20 mm de largo por 7-8 mm de ancho B. se deja unida por su base y se gira para suturarla al otro lado del nervio.

pero en algunos casos no se ha encontrado ninguna y en otros se han contabilizado dos o tres (a) Figura 5.

Antes del cierre de la herida se revisan y cauterizan las posibles bocas sangrantes, pese a lo cual se dejó en casi todos los casos una tira de plástico como drenaje antes de suturar el tejido celular subcutáneo. La piel se suturó con puntos sueltos de nylon monofilamento de $3 / 0$ ○ 4/0. La sutura se cubrió con una tira de Tulgrasum ${ }^{\circledR}$ antes de poner el vendaje compresivo desde la mitad del antebrazo a la mitad del brazo. En ningún caso se colocó férula u otra inmovilización en el codo, permitiendo al paciente que lo moviera y usara la mano desde el primer día hasta donde el dolor se lo permitiera.

\section{Hallazgos intraoperatorios:}

En todos los pacientes se anotó el aspecto macroscópico del nervio, registrándose la zona en la que éste

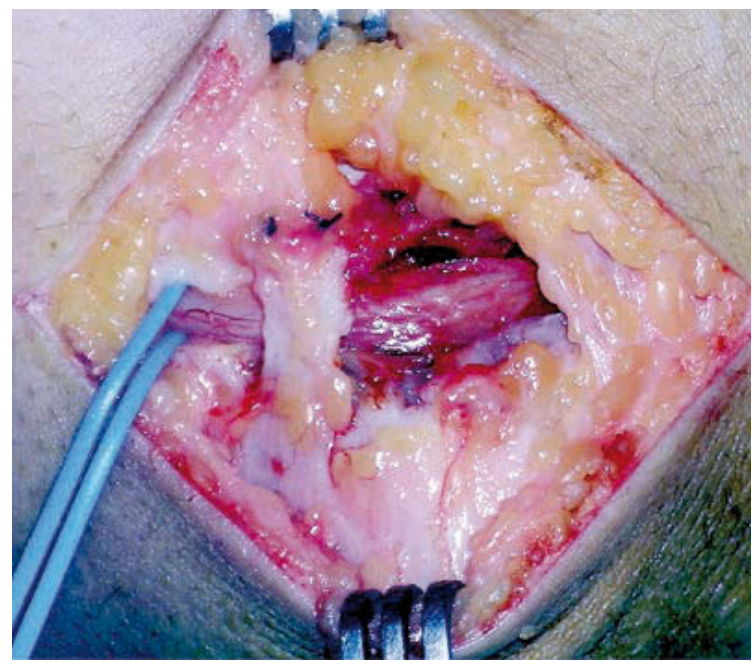

Figuras 4. Reconstrucción de posterior a anterior. se presentaba comprimido, así como la existencia de seudoneuroma, imagen en reloj de arena, cambios de coloración o alteraciones morfológicas de otro tipo.

\section{Valoración del estado postoperatorio y resultados clínicos:}

La valoración postoperatoria fue clínica (explorando fuerza y sensibilidad) y preguntando al paciente por su grado de satisfacción en cuanto a si notaba mejoría, estaba igual o había empeorado.

Para evaluar la evolución se utilizó una tabla comparativa entre el grado final con el previo en la escala de McGowan ${ }^{3}$, diferenciando cuatro posibles estados:

- Excelente: ganancia > 2 puntos; es decir, de 3 a I o de 2 a 0.

- Bueno: ganancia > I punto; de 3 a 2, 2 a I, o I a 0.

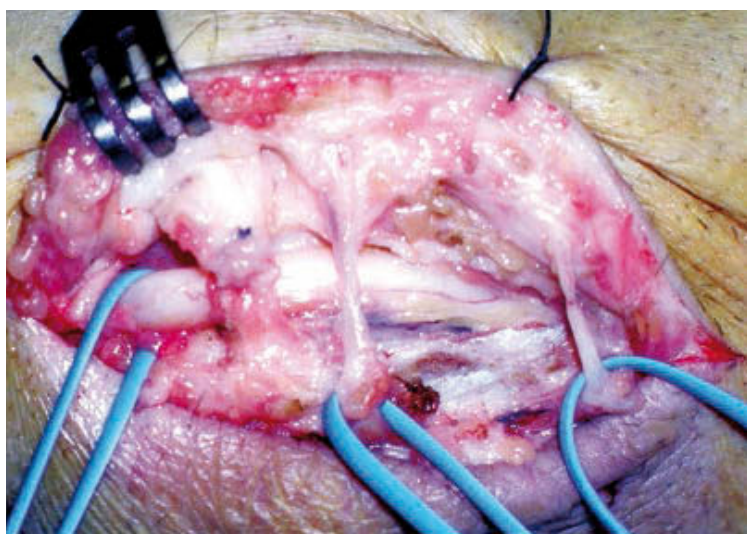

Figura 5.: Reconstrucción con dos bandeletas, una anterior, otra posterior, suturadas sobre el propio nervio con el nudo de sutura en el lado externo. Apréciense las dos ramas dorsales del nervio antebraquial cutáneo interno. 

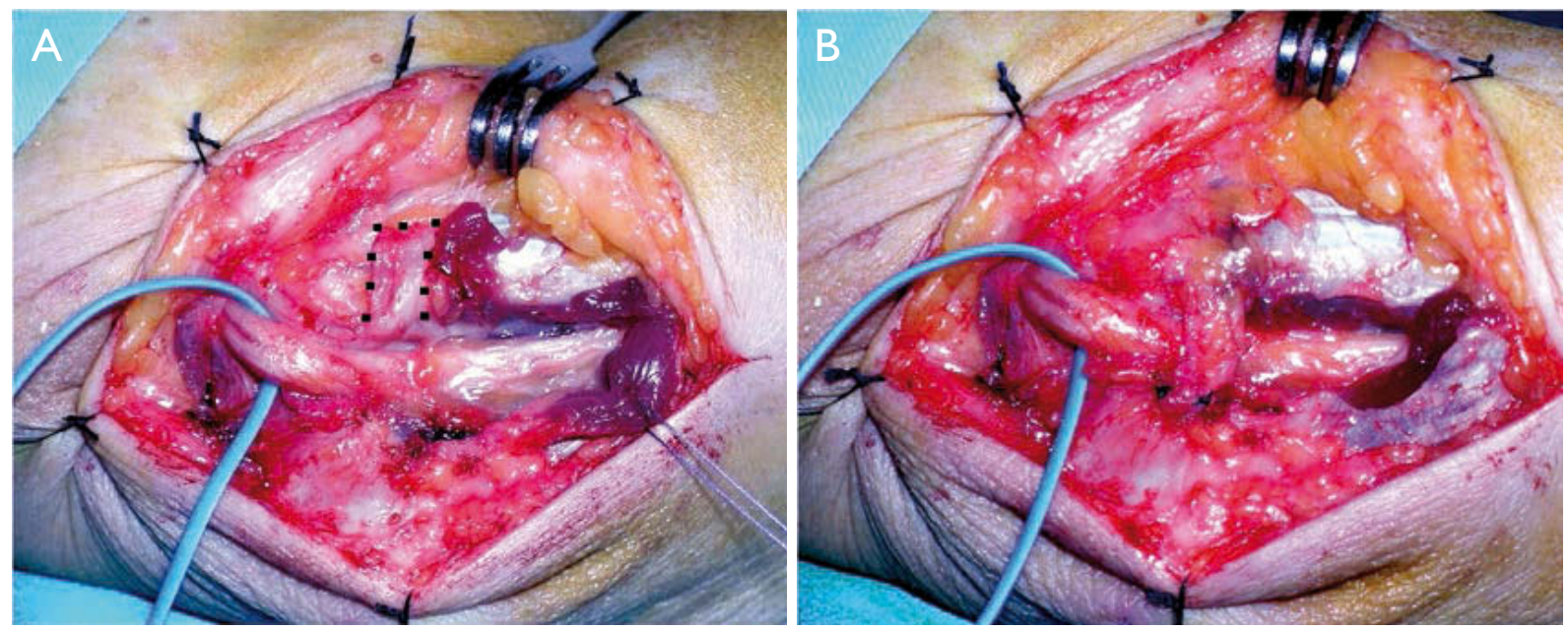

Figura 6. A. Desinserción del ligamento por su borde posterior, reflejado hacia delante para descomprimir el nervio, antes de ampliar su base. La línea de puntos enmarca su cara profunda. B. Polea ya suturada. Nótese la palidez del nervio en la zona distal y la resección de fibras musculares de la cabeza epitroclear del FCU.

- Regular: sin cambios: 3-3, 2-2, I-1.

- Malo: cuando hubo empeoramiento.

\section{RESULTADOS}

\section{Hallazgos intraoperatorios:}

El punto donde el nervio parecía más comprimido macroscópicamente fue bajo el ligamento epitrócleoolecraneano en 10 casos, bajo el FCU en 23, bajo ambos (ligamento y FCU) en 12, por tejido cicatricial en 4, e incierto, sin signos claros de compresión, en 2 codos en los que no se apreciaron cambios significativos en todo el trayecto disecado del nervio (los 2 con grado I), pese a lo cual mejoraron con la descompresión y reconstrucción de la nueva polea epitrócleo-olecraneana.

Un paciente varón, profesor de Educación Física, presentaba unos vasos sanguíneos muy dilatados que no parecían causantes ni coadyuvantes de la compresión sino un mero hallazgo intraoperatorio. Un paciente presentaba un músculo epitrocleocubital (epitrócleoancóneo, de la literatura sajona) bajo el cual estaba el punto de compresión (4) Figura 9 y en otra paciente se observó la existencia de fibras musculares en el ligamento epitrócleo-olecraneano (2) Figura 10; esta paciente tenía además muy desarrollado el vasto interno del tríceps, sin poder confirmar que éste fuera causa de la compresión.
Estado postoperatorio y resultados clínicos: No hubo infecciones de la herida, neuromas, ni rigidez del codo en ningún caso. Dos pacientes manifestaron hipoestesia temporalmente en el territorio cutáneo del NACl. Las molestias desaparecieron a las pocas semanas y en las últimas revisiones ninguna de ellas refirió trastornos sensitivos por este motivo. Un paciente tuvo una sección del NACl durante el abordaje y se realizó una neurorrafia.

De los II codos con grado I en 10 hubo una desaparición completa de la sintomatología y I manifestó haber mejorado, pero continuaba notando síntomas sensitivos ocasionalmente; es decir, en 10 pacientes se obtuvo un resultado excelente y en I bueno.

De los 28 codos con grado II la sintomatología desapareció en 22 y mejoró en 6 sin llegar a desaparecer completamente.

De los 12 codos con grado III el resultado fue excelente en 5 casos, (2 con recuperación motora y desaparición completa de la sintomatología, 3 con grado I de McGowan), bueno en 3 y regular en 4.

\section{El cómputo total figura reflejado en la (2) Tabla I.}

Todos los pacientes que se encontraban de baja laboral, se incorporaron a su actividad habitual con una media de 4I,5 días tras la intervención quirúrgica. 

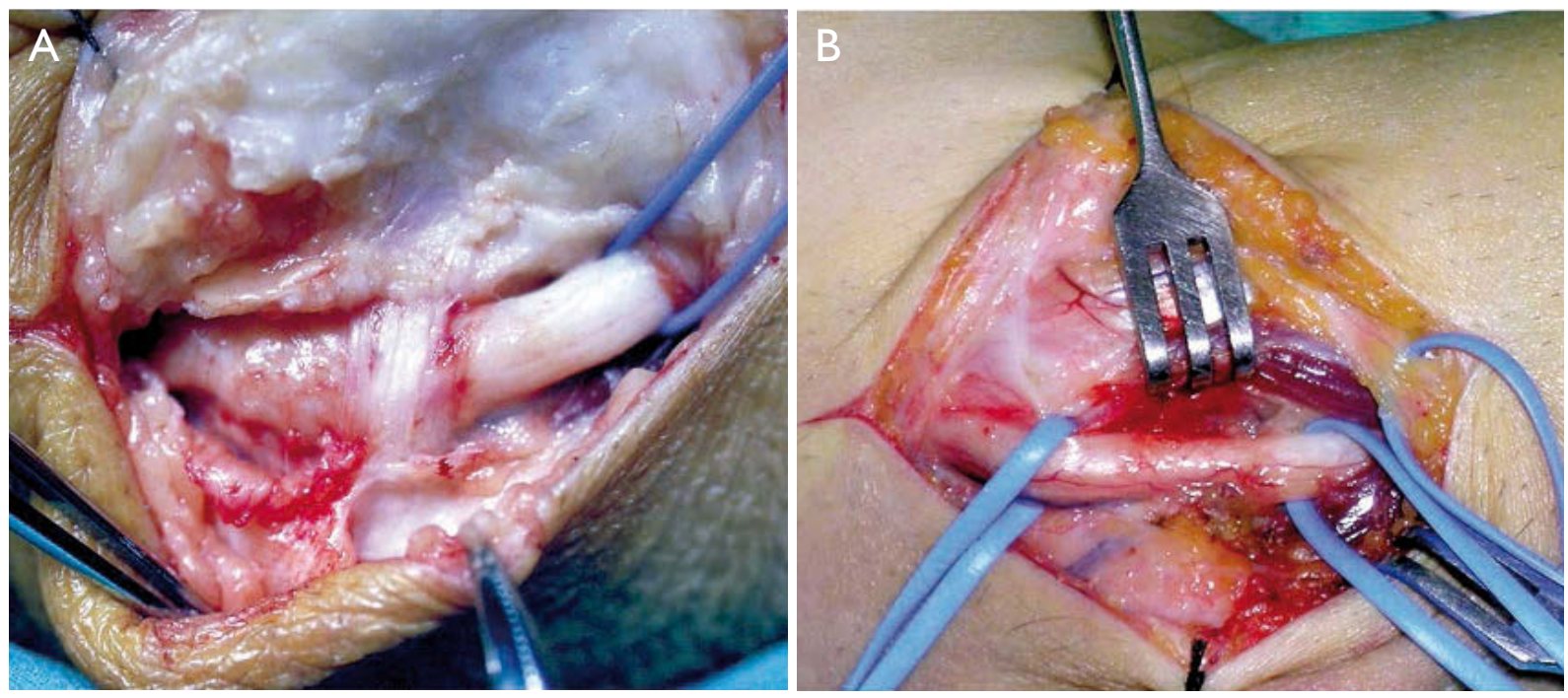

Figura 7. Cambios macroscópicos en el aspecto y coloración del nervio. A. Congestión e hiperemia en la zona proximal a la compresión nerviosa y palidez en la zona distal a la misma (vaseloop), donde se ve exangüe el vasa nervorum. B. Imagen en reloj de arena. El vasa nervorum se rellena al descomprimir el nervio, casi siempre en el curso de la intervención.

\section{DISCUSIÓN}

El tratamiento conservador del STCub está basado fundamentalmente en mantener el codo en semiextensión mediante el uso de ortesis nocturnas ${ }^{4,6}$. Su eficacia es escasa y está reservado a los casos de afectación leve o cuando hay contraindicaciones para la descompresión quirúrgica.

\begin{tabular}{c|c|c|c|c|c|}
$\begin{array}{c}\text { GRADO } \\
\text { PREOP. } \\
\text { Mc Gowan }\end{array}$ & N $^{\circ}$ & Excelente & Bueno & Regular & Malo \\
\hline I & II & 10 & I & 0 & 0 \\
\hline II & 28 & 22 & 6 & 0 & 0 \\
\hline III & 12 & 5 & 3 & 4 & 0 \\
\hline & 5 I & $37(72,6 \%)$ & $10(19,6 \%)$ & $4(7,8 \%)$ & 0 \\
\hline
\end{tabular}

Tabla. I.

El tratamiento quirúrgico comprende diversas técnicas diferentes, casi siempre descompresión simple, osteotomías epitrocleares o transposiciones anteriores. Los resultados de estas técnicas y las diversas modificaciones descritas en cada una de ellas muestran que aún se está lejos de haber llegado a un consenso sobre la técnica idónea.

Las transposiciones anteriores, bien subcutáneas o submusculares, tienen mayor morbilidad que la descompresión simple y no ofrecen ninguna ventaja, según se desprende de un amplio metaanálisis realizado por
Macadam y cols $^{7}$ tras comparar los resultados en 906 pacientes a quienes se les realizaron 449 descompresiones simples, 342 transposiciones subcutáneas y I 5 transposiciones submusculares sin hallar diferencias estadísticamente significativas. Diversos autores sostienen el mismo criterio: Brauer y Graham ${ }^{8}$ compararon las ventajas e inconvenientes de las cuatro modalidades de tratamiento en una amplia revisión bibliográfica llegando a la conclusión de que la mejor opción quirúrgica en las compresiones moderadas y severas es la simple descompresión. Chung ${ }^{9}$ admite haber abandonado su práctica de realizar transposiciones a favor de la descompresión simple. Bimmler y Meyer ${ }^{10}$, tras dividir a 79 pacientes en dos series de 31 con descompresión simple y 48 con transposición submuscular se muestran partidarios de la descompresión simple excepto en aquellos casos en los que aprecian tendencia a la subluxación tras la descompresión, en cuyo caso sí ven indicada la transposición anterior (conviene recordar que Childress" describió la existencia de subluxación o luxación habitual en el 16,2\% de la población general). Nabhan y cols ${ }^{12}$ en un estudio de 66 pacientes no encuentran diferencias significativas entre 32 descompresiones simples y 34 transposiciones anteriores subcutáneas, por lo que aconsejan la mera descompresión, así como tampoco encuentran diferencias Charles y cols ${ }^{13}$ en 49 pacientes tratados, 25 con transposición submuscular y 24 con transposición subcutánea.

En el presente estudio se exponen los datos de 51 codos operados con una técnica que evita la amplia 


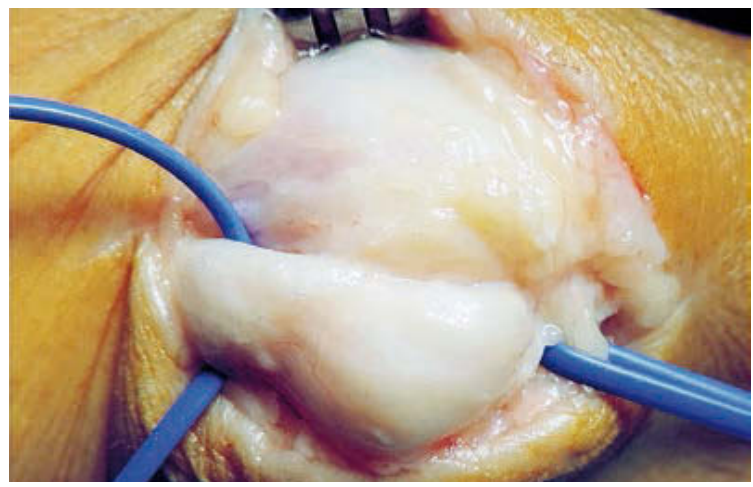

Figura 8. Seudoneuroma en una paciente añosa con McGowan grado III. El vaseloop de la izquierda rodea al nervio cubital; el de la derecha al nervio antebraquial cutáneo interno.

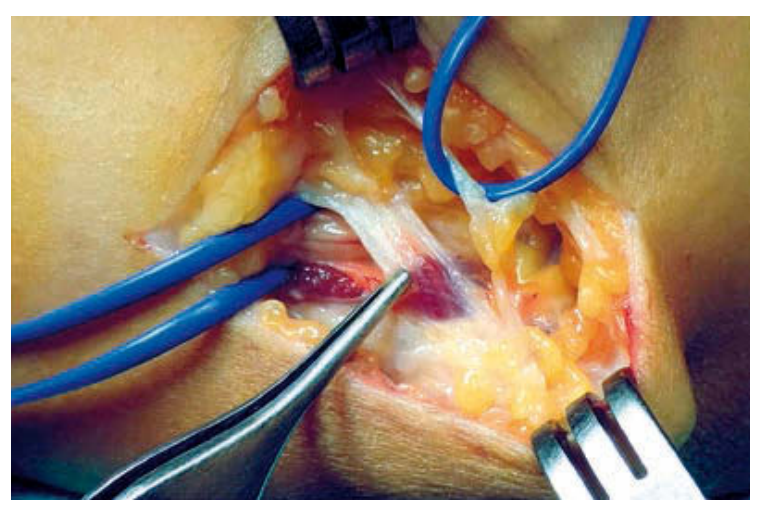

Figura | 0. Ligamento epitrócleo-olecraneano con fibras musculares (sujeto con una pinza de Adson).

disección del nervio propia de las transposiciones, con el consecuente deterioro de la vascularización neural, así como el riesgo de subluxación recurrente de las descompresiones simples y las complicaciones de las osteotomías epitrocleares (trastornos de la musculatura pronatoflexora, sangrado en el lecho nervioso con ulterior fibrosis perineural, etc.).

Algunos autores como Charles $^{13}$, Gelberman ${ }^{14}$ y Tan ${ }^{15}$ emplean una bandeleta similar a la descrita para impedir la movilización lateral del nervio, pero una vez hecha la transposición anterior. Rochet y cols ${ }^{16}$ reconstruyen la polea epitrócleo-olecraneana y dejan al nervio en su lecho natural, pero después de hacer una epitroclectomía.

La edad media fue muy similar a las series de Svernlöv ${ }^{6}$ (43 años) y Nathan ${ }^{17}$ (44,4 años) y algo menor a las de Charles' $^{13}$ (49,5 años), Kohut' ${ }^{18}$ (49 años y II meses), Rochet $^{16}$ (50,9 años), y Macadam (53 años), tal vez

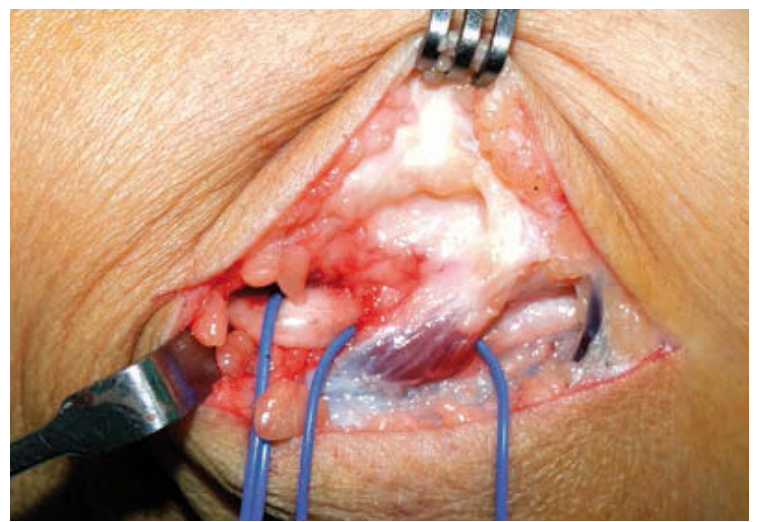

Figura 9. Músculo epitrocleocubital (epitrócleo-ancóneo, de la literatura sajona) bajo el cual estaba comprimido el nervio.

porque en este estudio buena parte de los pacientes (17; 34,7\%) provienen de una mutua de accidentes de trabajo; todos ellos, lógicamente, en edad laboral.

En nuestra serie el 96\% de los pacientes fueron diestros, cifra muy superior a la estimación del 10\% de zurdos en la población mundial'19.

En el $29,4 \%$ de los pacientes se operó el lado dominante y en el 70,6\% el lado no dominante, pero es arriesgado sacar conclusiones sobre la prevalencia de un lado u otro ya que en el ámbito laboral buena parte de los pacientes con afectación bilateral prefieren operar primero (o exclusivamente) el lado no dominante.

En esta serie de 5 I codos operados sólo hubo dos casos bilaterales (4\%). No abundan en la bibliografía médica los datos sobre la bilateralidad del STCub. En la serie de Nathan ${ }^{17}$ se operaron 102 casos en 74 pacientes, lo que representa $28(37,8 \%)$ cirugías bilaterales.

En general, el punto de compresión del nervio se manifiesta por cambios macroscópicos en el aspecto o la coloración. Es frecuente apreciar una notoria palidez del nervio en la zona de compresión, provocada por la isquemia ocasionada en el vasa nervorum, y una congestión e hiperemia en el área proximal a esa zona (1) Figura 7 ofreciendo a veces el nervio una imagen de seudoneuroma (2) Figura 8.

En esta serie la zona más frecuente de compresión se encontró en el tramo que transcurre desde el borde proximal del ligamento epitrócleo-olecraneano hasta 
el ángulo de unión de las dos cabezas proximales del FCU.

Se han descrito hasta 7 puntos de compresión en la proximidad del codo, que Siemionov ${ }^{20}$, tras su revisión bibliográfica, divide en tres localizaciones:

- Proximal al túnel cubital: en el septo intermuscular medial, arcada de Struthers o por el vasto interno del tríceps.

- En el túnel cubital: por el ligamento arqueado de Osborne o por el músculo epitrócleo-ancóneo.

- Distal al túnel cubital: entre las dos cabezas proximales del FCU o en el 1/3 proximal del antebrazo.

Aunque, como bien informó Osborne ${ }^{21}$, la diferenciación entre el ligamento epitrócleo-olecraneano y las dos inserciones proximales del FCU puede ser un tanto artificiosa, pues a menudo la fascia superficial que las une está fibrosada y hace indistinguible el inicio o final de ambas estructuras (-) Figura I.

Para Kohut ${ }^{18}$ no fue posible esclarecer el lugar exacto de la compresión en un $40 \%$ de sus pacientes, pero en la mayor parte de la literatura consultada no consta el lugar de compresión desde un punto de vista macroscópico, aspecto éste que puede ser muy importante para esclarecer la causa de la neuropatía.

La etiología del STCub se ha relacionado con múltiples causas y factores (disminución del calibre del canal, aumento de presión dentro del mismo, tracción, fricción o aumento de excursión del nervio, cambios isquémicos en el mismo, fibrosis cicatricial perineural, deformidades en varo o valgo del codo, etc.), pero, en general, el STCub idiopático se ha asociado con la flexión del codo prolongada o muy repetidal, 14,22, lo que explica la presencia de síntomas al despertarse en aquellas personas que tienen el hábito de dormir con los codos flexionados, y que todas las medidas del tratamiento conservador vayan encaminadas a prevenir la flexión del codo.

Son numerosos los artículos sobre mediciones de presión dentro del túnel| ${ }^{23-25}$ que demuestran un aumento con la flexión máxima del codo y su disminución con la extensión del mismo. Esto es debido a dos causas: por un lado, en la flexión, la epitróclea se separa del olécranon tensando así al ligamento epitrócleo-olecraneano, que comprime al nervio cubital (mecanismo compresivo), como demostraron los estudios de la
Clínica Mayo efectuados en cadáver por O’Driscoll122; por otro, al estar el nervio en la zona posterior de la interlínea articular, en la flexión del codo el nervio se estira al acodarse (mecanismo de tracción) ${ }^{14}$. Justamente este último mecanismo justifica el principio biomecánico en que se basan las transposiciones para pasar el nervio al plano anterior a la articulación. Sin embargo, es posible que este mecanismo de tracción sea menos importante que el de compresión por la distribución intraneural de las fibras nerviosas, que por enrollarse sobre sí mismas hacen al nervio más resistente a las fuerzas de tensión durante los movimientos de flexión del codo, al comportarse el nervio como una estructura espiral26.

Green y Rayan ${ }^{25}$ midieron la presión en el túnel cubital de 19 cadáveres, a $3 \mathrm{~cm}$ de la epitróclea en distintos grados de flexión, y observaron que la presión aumentaba a medida que flexionaba el codo y disminuía al cortar el ligamento epitrócleo-olecraneano.

Ochi y $\operatorname{cols}^{23}$ realizaron un test clínico preoperatorio de flexión del codo durante 45 segundos a 25 pacientes a los que luego, intraoperatoriamente, midieron la presión en el túnel cubital, encontrando que la presión aumentaba con la flexión del codo pero sin hallar correlación con el test clínico, que había sido positivo sólo en 12 de los 25 pacientes. De hecho, 2 de los 3 pacientes cuya presión extraneural había sido más alta, tenían un test de provocación con flexión del codo negativo. Dado lo cual, concluyen que la provocación de síntomas no se explica sólo por los cambios en la presión dinámica dentro del túnel y creen que debe haber otros factores.

Iba y cols $^{24}$, en cambio, sí hallan correlación significativa entre la presión extraneural dentro del túnel y la afectación clínica y electromiográfica. Al tiempo, confirman que la presión aumenta con la flexión del codo y el punto donde esta presión está más elevada la encuentran I cm distal al borde proximal del ligamento epitrócleo-olecraneano, descendiendo en sentido distal. Esta observación concuerda en parte con el trabajo previo de Green y Rayan ${ }^{25}$, quienes registraron la mayor presión $3 \mathrm{~cm}$ distales a la epitróclea, bajo el FCU, y no por debajo del ligamento epitrócleo-olecraneano.

En cualquier caso, sea cual sea la técnica quirúrgica elegida, parece que el factor terapéutico más importante es la sección del ligamento epitrócleo-olecraneano y la fascia de unión entre las dos cabezas del ECU. 
La descompresión simple in situ puede hacerse también con una pequeña incisión mediante endoscopia $^{27-30}$, pero esta técnica, al menos de momento, no permite reconstruir una nueva polea ni da una buena visión de las ramas sensitivas del $\mathrm{NACl}$.

Aunque la descompresión del nervio cubital, puede realizarse con anestesia local17,31, en nuestra serie no la hemos empleado en ningún caso por considerar que no ofrece ventajas significativas y sí varios inconvenientes: intolerancia al manguito de isquemia, posibilidad de dolor intraoperatorio, edematización de los tejidos que conlleva una peor visión de las estructuras anatómicas, así como mayor incomodidad y estrés para el cirujano.

De los 37 pacientes asegurados por cuenta ajena, 17 provenían de una mutua de accidentes de trabajo y todos estaban en situación de baja laboral, una buena parte en situación de enfermedad profesional. En España existe un vacío médico-legal con respecto a la consideración del trabajo como causa de STCub. Al contrario de lo que ocurre con el STC, donde parece clara la relación de ocupaciones de fuerza con el desarrollo de la enfermedad, algunos médicos laborales admiten el STCub como enfermedad profesional y otros no.

La comparación entre el estado previo y el postoperatorio requiere una clasificación inicial y ésta no es fácil en las patologías con gran componente subjetivo, como son las neuropatías periféricas especialmente en sus estados iniciales. La clasificación de McGowan ${ }^{3}$ no tiene en cuenta el grado de afectación electromiográfica, pero es una de las más extendida en la literatura médica y esto le confiere mayor facilidad de comparación bibliográfica.

El alivio de los síntomas resultó rápido en la mayoría de los pacientes, manifestando mejoría una gran parte de ellos ya en la primera semana de postoperatorio. Pero la desaparición completa de síntomas resultó lenta y se produjo de modo gradual a lo largo de varios meses. Lógicamente, en muy pocos casos con larga data de evolución y severa afectación motora y sensitiva (grado III) se produjo una recuperación completa de la atrofia muscular; sin embargo, en todos los casos hubo mejoría con respecto al estado preoperatorio.

Una de las complicaciones que encontramos en nuestra serie fue la lesión del $\mathrm{NACl}$ o las hipoestesias temporales en su territorio. Casi todos los autores de la literatura consultada obviaron esta complicación, por lo que apenas hay datos comparativos.
No se ha reintervenido a ningún paciente de esta serie por persistencia de síntomas. Bimmlerro realizó I I reintervenciones por recurrencia de la sintomatología en una serie de 79 pacientes; 9 de ellos después de una transposición anterior y 2 tras una descompresión simple.

En una serie de 69 codos operados, Goldfarb ${ }^{32}$ tuvo persistencia de síntomas en 5 (7\%) a los que les realizó una transposición anterior secundaria, pero todos ellos habían sido tratados inicialmente con una mera descompresión simple, sin reconstrucción de un nuevo retináculo.

\section{CONCLUSIONES}

La técnica descrita de descompresión simple con reconstrucción de un nuevo retináculo epitrócleo-olecraneano más elongado es una intervención quirúrgica que evita las posibles complicaciones de otras técnicas, como las amplias disecciones del nervio que exigen las transposiciones anteriores (lo que conllevará una menor devascularización y fibrosis perineural) y la morbilidad de las epitroclectomías en cuanto al sangrado en el lecho nervioso (fuente de fibrosis perineural). También impide la subluxación anterior propia de la descompresión simple.

La técnica propuesta está indicada en todos los grados de afectación siempre que la anatomía del codo sea normal y en pacientes que no fueron operados previamente con transposiciones anteriores, porque la reposición del nervio en su lugar de origen supondría una nueva agresión quirúrgica. Si hay un valgo, varo o alteraciones en el túnel por tumoraciones o antiguas lesiones, el tratamiento puede ser otro.

Todos los pacientes de nuestra serie se reincorporaron a su actividad habitual y tuvieron una mejoría clínica. Creemos, por consiguiente, que se trata de una técnica segura y con resultados predecibles.

\section{AGRADECIMIENTO}

A la Biblioteca del Complexo Hospitalario Universitario de A Coruña (CHUAC) por su valiosa colaboración en la búsqueda bibliográfica. 


\section{CONFLICTOS DE INTERESES}

Los autores declaran no tener conflictos de intereses.

\section{BIBLIOGRAFÍA}

I. Mackinnon SE, Novak CB. Neuropatías por compresión. En: Green DP, Hotchkiss RN, Pederson WC, Wolfe SW (Eds). Cirugía de la Mano. Madrid: Marbán Libros SL; 2007. p. 999-1033.

2. Panas J. Sur une cause peu connue de parálisis du nerf cubital. Arch Gen Med. I878;2:5-22.

3. Mc Gowan AJ.The results of transposition of the ulnar nerve for traumatic ulnar neuritis. J Bone Joint Surg Br. 1950;32-B(3):293-301.

4. Spinner M. Descompresión nerviosa. En: Morrey BF, (Ed). Codo "Master" en Cirugía Ortopédica. Madrid: Marbán Libros 2000. p. 183-97.

5. Testut L, Latarjet A.Tratado de anatomía humana. Ed. Barcelona: Salvat; 1975.

6. Svernlov B, Larsson M, Rehn K, Adolfsson L. Conservative treatment of the cubital tunnel syndrome. J Hand Surg EurVol. 2009;34(2):20 I-7.

7. Macadam SA, Gandhi R, Bezuhly M, Lefaivre KA. Simple decompression versus anterior subcutaneous and submuscular transposition of the ulnar nerve for cubital tunnel syndrome: a meta-analysis. J Hand Surg Am. 2008;33(8): 1314 el - 12.

8. Brauer CA, Graham B. The surgical treatment of cubital tunnel syndrome: a decision analysis. J Hand Surg EurVol. 2007;32(6):654-62.

9. Chung KC. Treatment of ulnar nerve compression at the elbow. J Hand Surg Am. 2008;33(9): 1625-7.

10. Bimmler D, Meyer VE. Surgical treatment of the ulnar nerve entrapment neuropathy: submuscular anterior transposition or simple decompression of the ulnar nerve? Long-term results in 79 cases. Ann Chir Main Memb Super. 1996; 15(3): 148-57.

I I. Childress HM. Recurrent ulnar-nerve dislocation at the elbow. J Bone Joint Surg Am. 1956;38-A(5): 978-84.

12. Nabhan A, Ahlhelm F, Kelm J, Reith W, Schwerdtfeger K, Steudel WI. Simple decompression or subcutaneous anterior transposition of the ulnar nerve for cubital tunnel syndrome. J Hand Surg Br. 2005;30(5):52I-4.
13. Charles YP, Coulet B, Rouzaud JC, Daures JP, Chammas M. Comparative clinical outcomes of submuscular and subcutaneous transposition of the ulnar nerve for cubital tunnel syndrome. J Hand Surg Am. 2009;34(5):866-74.

14. Gelberman RH, Eaton RG, Urbaniak JR. Peripheral nerve compression. Instr Course Lect. 1994; 43:31-53.

I5. Tan V, Pope J, Daluiski A, Capo JT, Weiland AJ. The V-sling: a modified medial intermuscular septal sling for anterior transposition of the ulnar nerve.J Hand Surg Am. 2004:29(2):325-7.

16. Rochet S, Obert L, Lepage D, Garbuio P, Tropet Y. Should we divide Osborn's ligament during epicondylectomy and in situ decompression of the ulnar nerve? Chir Main. 2004;23(3): 131-6.

17. Nathan PA, Istvan JA, Meadows KD. Intermediate and long-term outcomes following simple decompression of the ulnar nerve at the elbow. Chir Main. 2005;24(I):29-34.

18. Kohut GN, Della Santa DR, Chamay A. Ulnar nerve compression syndrome of the elbow. Analysis of 50 operated cases. Ann Chir Main Memb Super. 1996; 15(3): 138-47.

19. Hardyck C, Petrinovich LF. Left-handedness. Psychol Bull. 1977;84(3):385-404.

20. Siemionow M, Agaoglu G, Hoffmann R. Anatomic characteristics of a fascia and its bands overlying the ulnar nerve in the proximal forearm: a cadaver study. J Hand Surg EurVol. 2007;32(3):302-7.

21. Osborne GV. The surgical treatment of tardy ulnar neuritis. J Bone Joint Surg (Br). 1957;39B:782.

22. O'Driscoll SW, Horii E, Carmichael SW, Morrey BF. The cubital tunnel and ulnar neuropathy.J Bone Joint Surg Br. 1991;73(4):613-7.

23. Ochi K, Horiuchi Y, Nakamichi N, Morita K, Okada E, Hasegawa T. Association between the elbow flexion test and extraneural pressure inside the cubital tunnel. J Hand Surg Am. 2011;36(2): 216-21.

24. Iba K, Wada T, Aoki M,Tsuji H, Oda T,Yamashita T. Intraoperative measurement of pressure adjacent to the ulnar nerve in patients with cubital tunnel syndrome.J Hand Surg Am. 2006;3I (4):553-8.

25. Green JR, Jr., Rayan GM.The cubital tunnel: anatomic, histologic, and biomechanical study. J Shoulder Elbow Surg. 1999;8(5):466-70.

26. Llusa M, Mustafá-Gondolbeu A, León M, Morro R, Casañas J. Distribución intraneural del nervio cubital. Rev Esp Cir Ortop Traumatol. 20 I0;54(6):383-6. 
27. Cobb TK. Endoscopic cubital tunnel release. J Hand Surg Am. 20 I0;35(10):1690-7.

28. Hoffmann R, Siemionow M. The endoscopic management of cubital tunnel syndrome. J Hand Surg Br. 2006;31 ( I):23-9.

29. Mirza A, Reinhart MK, Bove J, Litwa J. Scope-assisted release of the cubital tunnel. J Hand Surg Am. 201 1;36(1):147-51.

30. Yoshida A, Okutsu I, Hamanaka I. Endoscopic anatomical nerve observation and minimally invasive management of cubital tunnel syndrome. J Hand Surg EurVol. 2009;34(I): I I 5-20.

31. Lankester B], Giddins GE. Ulnar nerve decompression in the cubital canal using local anaesthesia. J Hand Surg Br. 200 I;26(I):65-6.

32. Goldfarb CA, Sutter MM, Martens EJ, Manske PR. Incidence of re-operation and subjective outcome following in situ decompression of the ulnar nerve at the cubital tunnel. J Hand Surg Eur Vol. 2009;34(3):379-83. 